\title{
BERGMAN KERNEL ASYMPTOTICS AND A PURE ANALYTIC PROOF OF KODAIRA EMBEDDING THEOREM
}

\author{
CHIN-YU HSIAO
}

\begin{abstract}
In this paper, we survey recent results in [8] about the asymptotic expansion of Bergman kernel and we give a Bergman kernel proof of Kodaira embedding theorem.
\end{abstract}

\section{CONTENTS}

1. Introduction and Set up

1.1. Set up

2. Terminology in semi-classical analysis

3. Asymptotic expansion of Bergman kernel

3.1. Big line bundles and Shiffman conjecture

4. A Bergman kernel proof of Kodaira embedding theorem

References

\section{INTRODUCTION AND SET UP}

Let $L$ be a holomorphic line bundle over a complex manifold $M$ and let $L^{k}$ be the $k$-th tensor power of $L$. The Bergman projection $P_{k}$ is the orthogonal projection onto the space of $L^{2}$-integrable holomorphic sections of $L^{k}$. The study of the large $k$ behaviour of $P_{k}$ is an active research subject in complex geometry and is closely related to topics like the structure of algebraic manifolds, the existence of canonical Kähler metrics, Toeplitz quantization, equidistribution of zeros of holomorphic sections, quantum chaos and mathematical physics. We refer the reader to the book [11] for a comprehensive study of the Bergman kernel and its applications and also to the survey [10].

When $M$ is compact and $L$ is positive, Catlin [3] and Zelditch [13] established the asymptotic expansion of the Bergman kernel (see Theorem 4.2) by using a fundamental result by Boutet de Monvel-Sjöstrand [2] about the asymptotics of the Szegö kernel on a strictly pseudoconvex boundary. X. Dai, K. Liu and X. Ma [4] obtained the full off-diagonal asymptotic expansion and Agmon estimates of the Bergman kernel for a high power of positive line bundle on a compact complex manifold by using the heat kernel method. Ma and Marinescu [11], [12] proved the asymptotic expansion for yet another generalization of the Kodaira Laplacian, namely the renormalized Bochner-Laplacian on a symplectic manifold and also showed the existence of the estimate on a large class of non-compact manifolds. Another proof based on microlocal analysis of the existence of the full asymptotic expansion for the Bergman kernel for a high power of a positive line bundle on a compact complex manifold was obtained by Berndtsson, Berman and Sjöstrand [1].

In [8], we impose a very mild semiclassical local condition on $\bar{\partial}_{k}$, namely the $O\left(k^{-N}\right)$ small spectral gap on an open set $D \Subset M$ (see Definition 3.1), where $\bar{\partial}_{k}$ denotes the Cauchy-Riemann operator with values in $L^{k}$. We prove that the Bergman kernel admits an asymptotic expansion on $D$ if $\bar{\partial}_{k}$ has $O\left(k^{-N}\right)$ small spectral gap on $D$, cf. Theorem 3.2. Our approach bases on the microlocal Hodge

The author was partially supported by Taiwan Ministry of Science of Technology project 103-2115-M-001-001 and the Golden-Jade fellowship of Kenda Foundation. 
decomposition for Kohn Laplacian established in [7]. The distinctive feature of these asymptotics is that they work under minimal hypotheses. This allows us to apply them in situations which were up to now out of reach. We illustrate this in the study of the Bergman kernels of positive but singular Hermitian line bundles (see Theorem 3.5).

1.1. Set up. In this paper, we let $M$ be a not necessary compact complex manifold of dimension $n$ with a smooth positive $(1,1)$ form $\Theta$. $\Theta$ induces Hermitian metrics on the complexified tangent bundle $\mathbb{C} T M$ and $T^{* 0, q} M$ bundle of $(0, q)$ forms of $M, q=0,1, \ldots, n$. We shall denote all these Hermitian metrics by $\langle\cdot \mid \cdot\rangle$. Let $\left(L, h^{L}\right) \rightarrow M$ be a holomorpic line bundle over $M$, where $h^{L}$ denotes the Hermitian fiber metric of $L$. Let $R^{L}$ be the canonical curvature two form induced by $h^{L}$. Given a local trivializing section $s$ of $L$ on an open subset $D \subset M$ we define the associated local weight of $h^{L}$ by

$$
|s(x)|_{h^{L}}^{2}=e^{-2 \phi(x)}, \quad \phi \in C^{\infty}(D, \mathbb{R}) .
$$

Then $\left.R^{L}\right|_{D}=2 \partial \bar{\partial} \phi$. Let $\left(L^{k}, h^{L^{k}}\right)$ be the $k$-th tensor power of the line bundle $L$. If $s$ is a local trivializing section of $L,|s|_{h^{L}}^{2}=e^{-2 \phi}$, then $s^{k}$ is a local trivializing section of $L^{k}$ and $\left|s^{k}\right|_{h^{L^{k}}}^{2}=e^{-2 k \phi}$. We take $d v_{M}=d v_{M}(x)$ as the volume form on $M$ induced by $\Theta$. For every $q=0,1,2, \ldots, n$, let $(\cdot \mid \cdot)$ and $(\cdot \mid \cdot)_{h^{L^{k}}}$ be the standard $L^{2}$ inner products on $\Omega_{0}^{0, q}(M):=C_{0}^{\infty}\left(M, T^{* 0, q} M\right)$ and $\Omega_{0}^{0, q}\left(M, L^{k}\right):=$ $C_{0}^{\infty}\left(M, T^{* 0, q} M \otimes L^{k}\right)$ respectively induced by $d v_{M},\langle\cdot \mid \cdot\rangle$ and $h^{L^{k}}$ and we write $\|\cdot\|$ and $\|\cdot\|_{h^{L}}$ to denote the corresponding norms. Let $L_{(0, q)}^{2}(M)$ and $L_{(0, q)}^{2}\left(M, L^{k}\right)$ be the completions of $\Omega_{0}^{0, q}(M)$ and $\Omega_{0}^{0, q}\left(M, L^{k}\right)$ with respect to $\|\cdot\|$ and $\|\cdot\|_{h^{L^{k}}}$ respectively.

Let $\bar{\partial}_{k}: C^{\infty}\left(M, L^{k}\right) \rightarrow \Omega^{0,1}\left(M, L^{k}\right)$ be the Cauchy-Riemann operator with values in $L^{k}$. We extend $\bar{\partial}_{k}$ to $L^{2}\left(M, L^{k}\right):=L_{(0,0)}^{2}\left(M, L^{k}\right)$ by $\bar{\partial}_{k}: \operatorname{Dom} \bar{\partial}_{k} \subset L^{2}\left(M, L^{k}\right) \rightarrow L_{(0,1)}^{2}\left(M, L^{k}\right)$, where Dom $\bar{\partial}_{k}:=$ $\left\{u \in L^{2}\left(M, L^{k}\right) ; \bar{\partial}_{k} u \in L_{(0,1)}^{2}\left(M, L^{k}\right)\right\}$. Let

$$
P_{k}: L^{2}\left(M, L^{k}\right) \rightarrow \operatorname{Ker} \bar{\partial}_{k}
$$

be the Bergman projection, i.e. $P_{k}$ is the orthogonal projection onto $\operatorname{Ker} \bar{\partial}_{k}$ with respect to $(\cdot \mid \cdot)_{h^{L^{k}}}$ and let $P_{k}(x, y) \in C^{\infty}\left(M \times M, \mathscr{L}\left(L_{y}^{k}, L_{x}^{k}\right)\right)$ be the distribution kernel of $P_{k}$.

\section{TERMINOLOGY IN SEMI-CLASSICAL ANALYSIS}

In this section, we collect some definitions and notations in semi-classical analysis.

Let $B_{k}: L^{2}\left(M, L^{k}\right) \rightarrow L^{2}\left(M, L^{k}\right)$ be a continuous operator with smooth kernel $B_{k}(x, y)$. Let $s, s_{1}$ be local trivializing sections of $L$ on $D_{0} \Subset M, D_{1} \Subset M$ respectively, $|s|_{h^{L}}^{2}=e^{-2 \phi},\left|s_{1}\right|_{h^{L}}^{2}=e^{-2 \phi_{1}}$. The localized operator (with respect to the trivializing sections $s$ and $s_{1}$ ) of $B_{k}$ is given by

$$
\begin{aligned}
B_{k, s, s_{1}}: L_{\text {comp }}^{2}\left(D_{1}\right) & \rightarrow L^{2}(D), \\
u & \rightarrow e^{-k \phi^{-k}} B_{k}\left(s_{1}^{k} e^{k \phi_{1}} u\right) .
\end{aligned}
$$

and let $B_{k, s, s_{1}}(x, y) \in C^{\infty}\left(D \times D_{1}\right)$ be the distribution kernel of $B_{k, s, s_{1}}$, where

$$
L_{\text {comp }}^{2}\left(D_{1}\right):=\left\{v \in L^{2}\left(D_{1}\right) ; \operatorname{Supp} v \Subset D_{1}\right\} .
$$

Let $D$ be a local coordinate patch of $M$ and let $A_{k}: C_{0}^{\infty}(D) \rightarrow C^{\infty}(D)$ be a $k$-dependent continuous operator with smooth kernel $A_{k}(x, y)$. We write $A_{k} \equiv 0 \bmod O\left(k^{-\infty}\right)$ (on $D$ ) or $A_{k}(x, y) \equiv 0$ $\bmod O\left(k^{-\infty}\right)$ (on $D$ ) if $A_{k}(x, y)$ satisfies $\left|\partial_{x}^{\alpha} \partial_{y}^{\beta} A_{k}(x, y)\right|=O\left(k^{-N}\right)$ locally uniformly on every compact set in $D \times D$, for all multi-indices $\alpha, \beta \in \mathbb{N}^{2 n}$ and all $N>0$. Let $B_{k}: L^{2}\left(M, L^{k}\right) \rightarrow L^{2}\left(M, L^{k}\right)$ be a $k$-dependent continuous operator with smooth kernel. We write $B_{k} \equiv 0 \bmod O\left(k^{-\infty}\right)$ if $B_{k, s, s_{1}} \equiv 0$ $\bmod O\left(k^{-\infty}\right)$ for every local trivializing sections $s$ and $s_{1}$. 
Definition 2.1. Let $D$ be a local coordinate patch of $M$. Let $S(1 ; W)=S(1)$ be the set of all $a \in C^{\infty}(D)$ such that for every $\alpha \in \mathbb{N}^{2 n}$, there exists $C_{\alpha}>0$, such that $\left|\partial_{x}^{\alpha} a(x)\right| \leq C_{\alpha}$ on $W$. If $a=a(x, k)$ depends on $k \in] 1, \infty\left[\right.$, we say that $a(x, k) \in S_{\mathrm{loc}}(1 ; D)=S_{\mathrm{loc}}(1)$ if $\chi(x) a(x, k)$ uniformly bounded in $S(1)$ when $k$ varies in $] 1, \infty$ [, for any $\chi \in C_{0}^{\infty}(D)$. For $m \in \mathbb{R}$, we put $S_{\mathrm{loc}}^{m}(1 ; D)=S_{\mathrm{loc}}^{m}(1)=k^{m} S_{\mathrm{loc}}(1)$. If $a_{j} \in S_{\mathrm{loc}}^{m_{j}}(1), m_{j} \searrow-\infty$, we say that $a \sim \sum_{j=0}^{\infty} a_{j}$ (in $\left.S_{\mathrm{loc}}^{m_{0}}(1)\right)$ if $a-\sum_{j=0}^{N_{0}} a_{j} \in S_{\mathrm{loc}}^{m_{N_{0}+1}}(1)$ for every $N_{0}$. For a given sequence $a_{j}$ as above, we can always find such an asymptotic sum $a$ and $a$ is unique up to an element in $S_{\mathrm{loc}}^{-\infty}(1)=S_{\mathrm{loc}}^{-\infty}(1 ; W):=\bigcap_{m} S_{\mathrm{loc}}^{m}(1)$.

\section{ASYMPTOTIC EXPANSION OF BERGMAN KERNEL}

Let $s, s_{1}$ be local trivializing sections of $L$ on $D_{0} \Subset M, D_{1} \Subset M$ respectively, $|s|_{h^{L}}^{2}=e^{-2 \phi},\left|s_{1}\right|_{h^{L}}^{2}=$ $e^{-2 \phi_{1}}$. Let $P_{k, s, s_{1}}$ be the localized operator of $P_{k}$ given by (2.1) and let $P_{k, s, s_{1}}(x, y) \in C^{\infty}\left(D \times D_{1}\right)$ be the distribution kernel of $P_{k, s, s_{1}}$. When $s=s_{1}, D=D_{1}$, we write $P_{k, s}:=P_{k, s, s_{1}}, P_{k, s}(x, y):=$ $P_{k, s, s_{1}}(x, y)$. When $x=y, P_{k, s}(x, x)$ is independent of $s$. We write $P_{k}(x):=P_{k, s}(x, x)$ and we call $P_{k}(x)$ Bergman kernel function. Let $f_{1} \in C^{\infty}\left(M, L^{k}\right), \ldots, f_{d_{k}} \in C^{\infty}\left(M, L^{k}\right)$ be orthonormal frame for Ker $\bar{\partial}_{k}, d_{k} \in\{0\} \bigcup \mathbb{N} \bigcup\{\infty\}$. On $D_{0}$ and $D_{1}$, we write

$$
\begin{aligned}
& f_{j}=s^{k} e^{k \phi} \widetilde{f}_{j}, \quad \widetilde{f}_{j} \in C^{\infty}(D), \quad j=1,2, \ldots, d_{k}, \\
& f_{j}=s_{1}^{k} e^{k \phi_{1}} \hat{f}_{j}, \quad \hat{f}_{j} \in C^{\infty}\left(D_{1}\right), \quad j=1,2, \ldots, d_{k} .
\end{aligned}
$$

We can check that

$$
\begin{aligned}
& P_{k, s, s_{1}}(x, y)=\sum_{j=1}^{d_{k}} \widetilde{f}_{j}(x) \overline{\hat{f}_{j}}(y), \\
& P_{k}(x)=\sum_{j=1}^{d_{k}}\left|f_{j}(x)\right|_{h^{L^{k}}}^{2} .
\end{aligned}
$$

We recall $O\left(k^{-N}\right)$ small spectral gap property introduced in [8]

Definition 3.1. Let $D \subset M$. We say that $\bar{\partial}_{k}$ has $O\left(k^{-N}\right)$ small spectral gap on $D$ if there exist constants $C_{D}>0, N \in \mathbb{N}, k_{0} \in \mathbb{N}$, such that for all $k \geq k_{0}$ and $u \in C_{0}^{\infty}\left(D, L^{k}\right)$, we have

$$
\left\|\left(I-P_{k}\right) u\right\|_{h^{L^{k}}} \leq C_{D} k^{N}\left\|\bar{\partial}_{k} u\right\|_{h^{L^{k}}} .
$$

It should be mentioned that in [8], we actually introduced $O\left(k^{-N}\right)$ small spectral gap for Kodaira Laplacian. Note that $O\left(k^{-N}\right)$ small spectral gap for $\bar{\partial}_{k}$ implies $O\left(k^{-N}\right)$ small spectral gap for Kodaira Laplacian.

One of the main results in [8] is the following

Theorem 3.2. With the notations and assumptions used before, let $s$ be a local trivializing section of $L$ on an open set $D \subset M,|s|_{h^{L}}^{2}=e^{-2 \phi}$, and assume that $R^{L}$ is positive on $D$. Suppose that $\bar{\partial}_{k}$ has $O\left(k^{-N}\right)$ small spectral gap on $D$. Then, $\chi_{1} P_{k} \chi \equiv 0 \bmod O\left(k^{-\infty}\right)$ for every $\chi_{1} \in C_{0}^{\infty}(M), \chi \in C_{0}^{\infty}(D)$ with Supp $\chi_{1} \bigcap \operatorname{Supp} \chi=\emptyset$ and

$$
P_{k, s}(x, y) \equiv e^{i k \Psi(x, y)} b(x, y, k) \bmod O\left(k^{-\infty}\right) \text { on } D
$$

where $b(x, y, k) \sim \sum_{j=0}^{\infty} b_{j}(x, y) k^{n-j}$ in the sense of Definition 2.1. $b_{j}(x, y) \in C^{\infty}(D \times D), j=0,1, \ldots$, $b_{0}(x, x)=(2 \pi)^{-n}\left|\operatorname{det} R^{L}(x)\right|$ and

$$
\begin{aligned}
& \Psi(x, y) \in C^{\infty}(D \times D), \quad \Psi(x, y)=-\bar{\Psi}(y, x), \\
& \exists c>0: \operatorname{Im} \Psi \geq c|x-y|^{2}, \Psi(x, y)=0 \Leftrightarrow x=y,
\end{aligned}
$$


for any $p \in D$, take local holomorphic coordinates $z=\left(z_{1}, \ldots, z_{n}\right)$ vanishing at $p$, then near $(p, p)$,

$$
\Psi(z, w)=i(\phi(z)+\phi(w))-2 i \sum_{\alpha, \beta \in(\{0\} \cup \mathbb{N})^{n},|\alpha|+|\beta| \leq N} \frac{\partial^{|\alpha|+|\beta|} \phi}{\partial z^{\alpha} \partial \bar{z}^{\beta}}(0) \frac{z^{\alpha} \bar{w}^{\beta}}{\alpha ! \beta !}+O\left(|(z, w)|^{N+1}\right), \quad \forall N \in \mathbb{N},
$$

where $\operatorname{det} R^{L}(x)=\lambda_{1}(x) \cdots \lambda_{n}(x), \lambda_{j}(x), j=1, \ldots, n$, are the eigenvalues of $R^{L}$ with respect to $\langle\cdot \mid \cdot\rangle$.

In particular, $P_{k}(x) \sim \sum_{j=0}^{\infty} b_{j}(x, x) k^{n-j}$ in the sense of Definition 2.1

3.1. Big line bundles and Shiffman conjecture. As an application of Theorem 3.2 , we will establish Bergman kernel asymptotic expansion for big line bundle and this yields yet another proof of the Shiffman conjecture. Until further notice, we assume that $M$ is compact. We recall

Conjecture 3.3 (Shiffman, 1990). If $h^{L}$ is a singular Hermitian metric, smooth outside a proper analytic set $\Sigma, R^{L}>0$ in the sense of current, then $L$ is big.

$L$ is big if $\operatorname{dim} H^{0}\left(M, L^{k}\right) \approx k^{n}$, where $H^{0}\left(M, L^{k}\right)=\left\{u \in C^{\infty}\left(M, L^{k}\right) ; \bar{\partial}_{k} u=0\right\}$. Ji and Shiffman [9] solved this conjecture.

Now, we assume that $h^{L}$ is a singular Hermitian metric, smooth outside a proper analytic set $\Sigma$, $R^{L}>0$ in the sense of current. Consider the non-compact complex manifold $M \backslash \Sigma$. We also write $\bar{\partial}_{k}$ to denote the Cauchy-Riemann operator on $M \backslash \Sigma$ with values in $L^{k}$. Let $P_{k, M \backslash \Sigma}$ be the associated Bergman projection on $M \backslash \Sigma$ and let $P_{k, M \backslash \Sigma}(x)$ be the associated Bergman kernel function. In [8], we showed that

Theorem 3.4. $\bar{\partial}_{k}$ has $O\left(k^{-N}\right)$ small spectral gap on every $D \Subset M \backslash \Sigma$.

From Theorem 3.4 and Theorem 3.2, we deduce that

Theorem 3.5. $P_{k, M \backslash \Sigma}(x) \sim(2 \pi)^{-n}\left|\operatorname{det} R^{L}(x)\right| k^{n}+b_{1}(x) k^{n-1}+b_{2}(x) k^{n-2}+\cdots$ locally uniformly on $M \backslash \Sigma$, where $b_{j}(x) \in C^{\infty}(M \backslash \Sigma), j=1,2, \ldots$

Let $\left\{g_{1}, g_{2}, \ldots, g_{m_{k}}\right\}$ be an orthonormal frame for $H^{0}\left(M, L^{k}\right) \bigcap L^{2}\left(M \backslash \Sigma, L^{k}\right)$. The multiplier Bergman kernel function is defined by

$$
P_{k, \mathscr{I}}(x):=\sum_{j=1}^{m_{k}}\left|g_{j}(x)\right|_{h^{L^{k}}}^{2}, \quad x \in M \backslash \Sigma .
$$

The following result is essentially due o Skoda(see Demailly [5, Lemma 7.3, Ch. VIII]).

Theorem 3.6. $P_{k, M \backslash \Sigma}(x)=P_{k, \mathscr{I}}(x), \forall x \in M \backslash \Sigma$.

Proof of Shiffman conjecture. From Theorem 3.6 and Theorem 3.5, we establish Bergman kernel asymptotic expansion for big line bundle:

(3.4) $\quad P_{k, \mathscr{I}}(x) \sim(2 \pi)^{-n}\left|\operatorname{det} R^{L}(x)\right| k^{n}+b_{1}(x) k^{n-1}+b_{2}(x) k^{n-2}+\cdots$ locally uniformly on $M \backslash \Sigma$, where $b_{j}(x) \in C^{\infty}(M \backslash \Sigma), j=1,2, \ldots$. Let $K \Subset M \backslash \Sigma$. Note that $\operatorname{dim} H^{0}\left(M, L^{k}\right) \geq \int_{K} P_{k, \mathscr{I}}(x) d v_{M}(x)$. From this observation and (3.4), we reprove Shiffman conjecture.

\section{A BERGMAN KERNEL PROOF OF KodAIRA EMBEDDING THEOREM}

For a holomorphic line bundle $E \rightarrow M$, we say that $E$ is positive if there is a Hermitian metric $h^{E}$ of $E$ such that the associated curvature $R^{E}$ is positive definite on $M$. Let's recall Kodaira embedding theorem first.

Theorem 4.1. Let $M$ be a compact complex manifold. If there is a positive holomorphic line bundle $E$ over $M$, then $M$ can be holomorphic embedded into $\mathbb{C P}^{N}$, for some $N \in \mathbb{N}$. 
We return to our situation and we will use the same notations as before. By using Hörmander's $L^{2}$ estimates [6], it is easy to see that if $M$ is compact and $R^{L}$ is positive on $M$ then $\bar{\partial}_{k}$ has $O\left(k^{-N}\right)$ small spectral gap on $M$. From this observation and Theorem 3.2, we deduce

Theorem 4.2. Assume that $M$ is compact and $R^{L}$ is positive on $M$. Then,

$$
\chi_{1} P_{k} \chi \equiv 0 \quad \bmod O\left(k^{-\infty}\right)
$$

for every $\chi_{1} \in C^{\infty}(M), \chi \in C^{\infty}(M)$ with $\operatorname{Supp} \chi_{1} \bigcap \operatorname{Supp} \chi=\emptyset$. Let $s$ be a local trivializing section of $L$ on an open set $D \subset M,|s|_{h^{L}}^{2}=e^{-2 \phi}$, then

$$
P_{k, s}(x, y) \equiv e^{i k \Psi(x, y)} b(x, y, k) \bmod O\left(k^{-\infty}\right) \text { on } D,
$$

where $b(x, y, k)$ and $\Psi(x, y)$ are as in Theorem 3.2

In particular,

$$
P_{k}(x) \sim(2 \pi)^{-n}\left|\operatorname{det} R^{L}(x)\right| k^{n}+b_{1}(x) k^{n-1}+b_{2}(x) k^{n-2}+\cdots \text { uniformly on } M .
$$

By using Theorem 3.2, we are going to give a Bergman kernel proof of Kodaira embedding theorem. From now on, we assume that $R^{L}$ is positive on $M$. As before, put

$$
H^{0}\left(M, L^{k}\right):=\left\{u \in C^{\infty}\left(M, L^{k}\right) ; \bar{\partial}_{k} u=0\right\}
$$

and let $\left\{f_{1}, \ldots, f_{d_{k}}\right\}$ be an orthonormal basis for $H^{0}\left(M, L^{k}\right)$ with respect to $(\cdot \mid \cdot)_{h^{L^{k}}}$. The Kodaira map is given by

$$
\Phi_{k}: x \in X \rightarrow\left[f_{1}(x), f_{2}(x), \ldots, f_{d_{k}}(x)\right] \in \mathbb{C P}^{d_{k}-1}
$$

From (4.3), we see that there is a $k_{0}>0$ such that for every $k \geq k_{0}, \sum_{j=1}^{d_{k}}\left|f_{j}(x)\right|_{h^{L}}^{2} \geq c k^{n}$ on $M$, where $c>0$ is a constant independent of $k$. Hence, fix any $k \geq k_{0}$, for every $x \in X$, there is a $f_{j}$, $j \in\left\{1,2, \ldots, d_{k}\right\}$, such that $\left|f_{j}(x)\right|_{h^{L^{k}}}^{2}>0$. We conclude that $\Phi_{k}$ is a well-defined as a smooth map from $X$ to $\mathbb{C P}^{d_{k}-1}$. We will prove

Theorem 4.3. For $k$ large, $\Phi_{k}$ is a holomorphic embedding.

It is clearly that Kodaira embedding theorem follows from Theorem 4.3 . We recall that for a smooth map $\Phi: X \rightarrow \mathbb{C P}^{N}$ is an embedding if $d \Phi_{x}: T X \rightarrow T \mathbb{C P}^{N}$ is injective at each point $x \in X$ and $\Phi: X \rightarrow \mathbb{C P}^{N}$ is globally injective.

Let $s$ be a local trivializing section of $L$ on an open set $D \subset M$. Fix $p \in D$ and let $z=\left(z_{1}, \ldots, z_{n}\right)=$ $x=\left(x_{1}, \ldots, x_{2 n}\right), z_{j}=x_{2 j-1}+i x_{2 j}, j=1, \ldots, n$, be local holomorphic coordinates of $X$ defined in some small neighbourhood of $p$ such that

$$
\phi(z)=\sum_{j=1}^{n} \lambda_{j}\left|z_{j}\right|^{2}+O\left(|z|^{3}\right),
$$

where $2 \lambda_{1}, \ldots, 2 \lambda_{n}$ are the eigenvalues of $R^{L}(p)$ with respect to $\langle\cdot \mid \cdot\rangle$. We may assume that the local coordinates $z$ defined on $D$. We also write $y=\left(y_{1}, \ldots, y_{2 n}\right)$. Until further notice, we work on $D$. Take $\chi \in C_{0}^{\infty}(\mathbb{R},[0,1])$ with $\chi(x)=1$ on $\left[-\frac{1}{2}, \frac{1}{2}\right], \chi(x)=0$ on $\left.]-\infty,-1\right] \cup[1, \infty[$ and $\chi(t)=\chi(-t)$, for every $t \in \mathbb{R}$. Let

$$
u_{k}:=P_{k}\left(s^{k} e^{k \phi} \chi\left(\sqrt{k} y_{1}\right) \cdots \chi\left(\sqrt{k} y_{2 n}\right)\right) \in H^{0}\left(M, L^{k}\right) .
$$

On $D$, we write $u_{k}=s^{k} e^{k \phi} \widetilde{u}_{k}, \widetilde{u}_{k} \in C^{\infty}(D)$. Then, $\left|u_{k}(x)\right|_{h^{L^{k}}}^{2}=\left|\widetilde{u}_{k}(x)\right|^{2}, \forall x \in D$. We need

Lemma 4.4. With the notations used above, there is a $k_{0}>0$ independent of $k$ and the point $p$ such that for all $k \geq k_{0}$,

$$
\left|u_{k}(p)\right|_{h^{L^{k}}}^{2} \geq c_{0}
$$




$$
\left|u_{k}(x)\right|_{h^{L^{k}}}^{2} \leq \frac{1}{c_{0} k}, \quad \forall x \notin D
$$

and

$$
\left|\frac{1}{\sqrt{k}} \frac{\partial \widetilde{u}_{k}}{\partial x_{s}}(p)\right| \leq \frac{1}{c_{0} k}, \quad s=1,2, \ldots, 2 n,
$$

where $c_{0}>0$ is a constant independent of $k$ and the point $p$.

Proof. From (4.2), we can check that

$$
\begin{aligned}
& \widetilde{u}_{k}(x) \\
& \equiv \int e^{i k \Psi(x, y)} b(x, y, k) \chi\left(\sqrt{k} y_{1}\right) \cdots \chi\left(\sqrt{k} y_{2 n}\right) d v_{M}(y) \bmod O\left(k^{-\infty}\right) \\
& \equiv \int e^{i k \Psi\left(x, \frac{y}{\sqrt{k}}\right)} k^{-n} b\left(x, \frac{y}{\sqrt{k}}, k\right) \chi\left(y_{1}\right) \cdots \chi\left(y_{2 n}\right) d v_{M}(y) \bmod O\left(k^{-\infty}\right) .
\end{aligned}
$$

From (3.3), Theorem 4.2 and note that $\Psi(0,0)=0$, we can check that

$$
\lim _{k \rightarrow \infty} \widetilde{u}_{k}(p)=\frac{1}{2} \pi^{-n}\left|\operatorname{det} R_{p}^{L}\right| \int \chi\left(y_{1}\right) \cdots \chi\left(y_{2 n}\right) d v_{M}(y) .
$$

Similarly, it is straightforward to check that $\lim _{k \rightarrow \infty} \frac{1}{\sqrt{k}} \frac{\partial \widetilde{u}_{k}}{\partial x_{s}}(p)=0, s=1,2, \ldots, 2 n$. Hence, there is a constant $\widetilde{k}_{0}>0$ such that for every $k \geq \widetilde{k}_{0}$, (4.7) and (4.9) hold. Since $X$ is compact, $\widetilde{k}_{0}$ can be taken to be independent of the point $p$.

Now, we prove (4.8). Since $x \notin D$, from (4.1), we see that $\left|u_{k}(x)\right|_{h^{L^{k}}}^{2} \equiv 0 \bmod O\left(k^{-\infty}\right)$ outside $D$. Thus, there is a constant $\hat{k}_{0}>0$ such that for every $k \geq \hat{k}_{0}$, (4.8) holds. Since $X$ is compact, $\hat{k}_{0}$ can be taken to be independent of the point $p$. The lemma follows.

For every $j=1,2, \ldots, n$, let

$$
u_{k}^{j}:=P_{k}\left(s^{k} e^{k \phi} \sqrt{k}\left(y_{2 j-1}+i y_{2 j}\right) \chi\left(\sqrt{k} y_{1}\right) \cdots \chi\left(\sqrt{k} y_{2 n}\right)\right) \in H^{0}\left(M, L^{k}\right) .
$$

On $D$, we write $u_{k}^{j}=s^{k} e^{k \phi} \widetilde{u}_{k}^{j}, \widetilde{u}_{k}^{j} \in C^{\infty}(D), j=1,2, \ldots, n$. The following follows from some straightforward computation and essentially the same as the proof of Lemma 4.4. We omit the details.

Lemma 4.5. With the notations used above, there is a $k_{1}>0$ independent of $k$ and the point $p$ such that for all $k \geq k_{1}$,

$$
\begin{aligned}
& \left|\widetilde{u}_{k}^{j}(p)\right| \leq \frac{1}{c_{1} k}, \quad j=1,2, \ldots, n, \quad\left|\frac{1}{\sqrt{k}} \frac{\partial \widetilde{u}_{k}^{j}}{\partial \bar{z}_{s}}(p)\right| \leq \frac{1}{c_{1} k}, \quad j, s=1,2, \ldots, n, \\
& \left|\frac{1}{\sqrt{k}} \frac{\partial \widetilde{u}_{k}^{j}}{\partial z_{s}}(p)\right| \leq \frac{1}{c_{1} k}, \quad j, s=1,2, \ldots, n-1, \quad j \neq s, \quad\left|\frac{1}{\sqrt{k}} \frac{\partial \widetilde{u}_{k}^{j}}{\partial z_{j}}(p)\right| \geq c_{1}, \quad j=1,2, \ldots, n,
\end{aligned}
$$

where $c_{1}>0$ is a constant independent of $k$ and the point $p$.

From now on, we take $k$ be a large constant so that $k>>2\left(k_{0}+k_{1}\right)$, where $k_{0}>0$ and $k_{1}>0$ are constants as in Lemma 4.4 and Lemma 4.5, We can prove

Theorem 4.6. $d \Phi_{k}(x): T_{x} X \rightarrow T_{x} \mathbb{C P}^{d_{k}-1}$ is injective at every $x \in X$.

Proof. Fix $p \in X$ and let $s$ be a local trivializing section of $L$ on an open set $D \subset M, p \in D$. Let $u_{k} \in H^{0}\left(M, L^{k}\right)$ and $u_{k}^{j} \in H^{0}\left(M, L^{k}\right), j=1,2, \ldots, n$, be as in Lemma 4.4 and Lemma 4.5. From Lemma 4.4 and Lemma 4.5, it is not difficult to check that $u_{k}, u_{k}^{1}, u_{k}^{2} \ldots, u_{k}^{n}$ are linearly independent. Take $\left\{u_{k}, u_{k}^{1}, u_{k}^{2}, \ldots, u_{k}^{n}, g_{1}, \ldots, g_{m_{k}}\right\}$ be a basis (not orthogonal) for $H^{0}\left(M, L^{k}\right), m_{k}=d_{k}-n-1$. From Lemma 4.4 and Lemma 4.5 , it is easy to see that

$$
\text { the differential of the map } x \rightarrow\left(\frac{u_{k}^{1}}{u_{k}}, \ldots, \frac{u_{k}^{n}}{u_{k}}, \frac{g_{1}}{u_{k}}, \ldots, \frac{g_{m_{k}}}{u_{k}}\right) \text { is injective at } p \text {. }
$$


From (4.13) and some elementary linear algebra, it is not difficult to check that $d \Phi_{k}(p): T_{p} X \rightarrow$ $T_{p} \mathbb{C P}^{d_{k}-1}$ is injective. We omit the detail.

Now, we can prove

Theorem 4.7. For $k$ large, $\Phi_{k}: X \rightarrow \mathbb{C P}^{d_{k}-1}$ is globally injective.

Proof. We assume that the claim of the theorem is not true. We can find $x_{k_{j}}, y_{k_{j}} \in M, x_{k_{j}} \neq y_{k_{j}}$, $0<k_{1}<k_{2}<\cdots, \lim _{j \rightarrow \infty} k_{j}=\infty$, such that $\Phi_{k_{j}}\left(x_{k_{j}}\right)=\Phi_{k_{j}}\left(y_{k_{j}}\right)$, for each $j$. We may suppose that there are $x_{k}, y_{k} \in M, x_{k} \neq y_{k}$, such that $\Phi_{k}\left(x_{k}\right)=\Phi_{k}\left(y_{k}\right)$, for each $k$. Thus, $\left[f_{1}\left(x_{k}\right), \cdots, f_{d_{k}}\left(x_{k}\right)\right]=$ $\left[f_{1}\left(y_{k}\right), \cdots, f_{d_{k}}\left(y_{k}\right)\right]$, for each $k$. We conclude that for every $g_{k} \in H^{0}\left(M, L^{k}\right)$, there is a $\lambda_{k} \in \mathbb{C}$ such that

$$
g_{k}\left(x_{k}\right)=\lambda_{k} g_{k}\left(y_{k}\right)
$$

We may assume that $\left|\lambda_{k}\right| \geq 1$. Hence, for every $g_{k} \in H^{0}\left(M, L^{k}\right)$,

$$
\left|g_{k}\left(x_{k}\right)\right|_{h^{L^{k}}}^{2} \geq\left|g_{k}\left(y_{k}\right)\right|_{h^{L^{k}}}^{2} .
$$

Since $M$ is compact, we may assume that $x_{k} \rightarrow p \in M, y_{k} \rightarrow q \in M$, as $k \rightarrow \infty$. Suppose that $p \neq q$. In view of Lemma 4.4, we see that there is a $v_{k} \in H^{0}\left(M, L^{k}\right)$ with $\left|v_{k}\left(y_{k}\right)\right|_{h^{L^{k}}}^{2} \geq c_{0}$ and $\left|v_{k}\left(x_{k}\right)\right|_{h^{L^{k}}}^{2} \leq$ $\frac{1}{c_{0} k}$, where $c_{0}>0$ is a constant independent of $k$. Thus, for $k$ large, $\left|v_{k}\left(x_{k}\right)\right|_{h^{L^{k}}}^{2}<\left|v_{k}\left(y_{k}\right)\right|_{h^{L^{k}}}^{2}$. From this and (4.15), we get a contradiction. Thus, we must have $p=q$.

Let $s$ be a local trivializing section of $L$ on an open subset $D \subset X$ of $p,|s|_{h^{L}}^{2}=e^{-2 \phi}$. Now, we assume that $x_{k} \rightarrow p \in M, y_{k} \rightarrow p \in M$, as $k \rightarrow \infty$. Let $z=\left(z_{1}, \ldots, z_{n}\right)=x=\left(x_{1}, \ldots, x_{2 n}\right), z_{j}=x_{2 j-1}+i x_{2 j}$, $j=1, \ldots, n$, be local holomorphic coordinates of $X$ defined in some small neighbourhood of $p$ such that (4.5) hold. We may assume that $x_{k}, y_{k} \in D$ for each $k$ and the local coordinates $x$ defined on $D$. We shall use the same notations as before.

Case I : $\lim _{\sup _{k \rightarrow \infty}} \sqrt{k}\left|x_{k}-y_{k}\right|=M>0(M$ can be $\infty)$.

For simplicity, we may assume that

$$
\left.\left.\lim _{k \rightarrow \infty} \sqrt{k}\left|x_{k}-y_{k}\right|=M, \quad M \in\right] 0, \infty\right] .
$$

On $D$, we write $f_{j}=s^{k} e^{k \phi} \widetilde{f}_{j}, \widetilde{f}_{j} \in C^{\infty}(D), j=1, \ldots, d_{k}$. Put

$$
v_{k}(x):=\sum_{j=1}^{d_{k}} f_{j}(x) \overline{\widetilde{f}_{j}\left(y_{k}\right)} \in H^{0}\left(M, L^{k}\right) .
$$

We can check that

$$
\begin{aligned}
\left|v_{k}\left(x_{k}\right)\right|_{h^{L} k}^{2} & =\left|\sum_{j=1}^{d_{k}} \widetilde{f}_{j}\left(x_{k}\right) \overline{\widetilde{f}_{j}\left(y_{k}\right)}\right|^{2}=\left|P_{k, s}\left(x_{k}, y_{k}\right)\right|^{2}=\left|e^{i k \Psi\left(x_{k}, y_{k}\right)} b\left(x_{k}, y_{k}, k\right)\right|^{2} \\
& \leq e^{-2 k \operatorname{Im} \Psi\left(x_{k}, y_{k}\right)}\left|b\left(x_{k}, y_{k}, k\right)\right|^{2}
\end{aligned}
$$

and

$$
\left|v_{k}\left(y_{k}\right)\right|_{h^{L^{k}}}^{2}=\left|P_{k, s}\left(y_{k}, y_{k}\right)\right|^{2}=\left|e^{i k \Psi\left(y_{k}, y_{k}\right)} b\left(y_{k}, y_{k}, k\right)\right|^{2}=\left|b\left(y_{k}, y_{k}, k\right)\right|^{2} .
$$

From the fact that $\operatorname{Im} \Psi(x, y) \geq c|x-y|^{2}$, where $c>0$ is a constant, (4.16), (4.18) and (4.19), we can check that

$$
\lim _{k \rightarrow \infty} k^{-2 n}\left|v_{k}\left(x_{k}\right)\right|_{h^{L^{k}}}^{2} \leq e^{-2 c M^{2}}\left|b_{0}(p, p)\right|^{2}<\left|b_{0}(p, p)\right|^{2}=\lim _{k \rightarrow \infty} k^{-2 n}\left|v_{k}\left(y_{k}\right)\right|_{h^{L^{k}}}^{2},
$$

where $b_{0}$ is the leading term of $b(x, y, k)$. Note that $b_{0}(p, p)=(2 \pi)^{-n}\left|\operatorname{det} R^{L}(p)\right|>0$ (see Theorem 3.2). From (4.20) and (4.15), we get a contradiction.

Case II : $\lim _{\sup _{k \rightarrow \infty}} \sqrt{k}\left|x_{k}-y_{k}\right|=0$. 
Put $f_{k}(t)=\frac{\left|v_{k}\left(t x_{k}+(1-t) y_{k}\right)\right|_{h^{k}}^{2}}{P_{k}\left(t x_{k}+(1-t) y_{k}\right) P_{k}\left(y_{k}\right)}$, where $v_{k}$ is as in (4.17). We can check that

$$
f_{k}(t)=\frac{\left|\sum_{j=1}^{d_{k}} \widetilde{f}_{j}\left(t x_{k}+(1-t) y_{k}\right) \overline{\widetilde{f}_{j}\left(y_{k}\right)}\right|^{2}}{\sum_{j=1}^{d_{k}}\left|\widetilde{f}_{j}\left(t x_{k}+(1-t) y_{k}\right)\right|^{2} \sum_{j=1}^{d_{k}}\left|\widetilde{f}_{j}\left(y_{k}\right)\right|^{2}}=\frac{\left|P_{k, s}\left(t x_{k}+(1-t) y_{k}, y_{k}\right)\right|^{2}}{P_{k}\left(t x_{k}+(1-t) y_{k}\right) P_{k}\left(y_{k}\right)} .
$$

From (4.14) and (4.21), it is easy to see that $0 \leq f_{k}(t) \leq 1, \forall t \in[0,1]$ and $f_{k}(0)=f_{k}(1)=1$. Thus, for each $k$, there is a $t_{k} \in[0,1]$ such that $f_{k}^{\prime \prime}\left(t_{k}\right) \geq 0$. Hence,

$$
\liminf _{k \rightarrow \infty} \frac{f_{k}^{\prime \prime}\left(t_{k}\right)}{\left|x_{k}-y_{k}\right|^{2} k} \geq 0
$$

From (4.2), we see that

$$
\begin{aligned}
& \left|P_{k, s}\left(t x_{k}+(1-t) y_{k}, y_{k}\right)\right|^{2}=e^{-2 k \operatorname{Im} \Psi\left(t x_{k}+(1-t) y_{k}, y_{k}\right)}\left|b\left(t x_{k}+(1-t) y_{k}, y_{k}, k\right)\right|^{2}, \\
& P_{k}\left(t x_{k}+(1-t) y_{k}\right) \\
& =b\left(t x_{k}+(1-t) y_{k}, t x_{k}+(1-t) y_{k}, k\right) \sim \sum_{j=0}^{\infty} k^{n-j} b_{j}\left(t x_{k}+(1-t) y_{k}, t x_{k}+(1-t) y_{k}\right) .
\end{aligned}
$$

From (4.23), it is straightforward to calculate that

(4.24)

$$
\begin{aligned}
& \frac{\partial\left|P_{k, s}\left(t x_{k}+(1-t) y_{k}, y_{k}\right)\right|}{\partial t} \\
& =e^{-2 k \operatorname{Im} \Psi\left(t x_{k}+(1-t) y_{k}, y_{k}\right)}\left(\left\langle-2 k \operatorname{Im} \Psi_{x}^{\prime}\left(t x_{k}+(1-t) y_{k}, y_{k}\right), x_{k}-y_{k}\right\rangle\left|b\left(t x_{k}+(1-t) y_{k}, y_{k}, k\right)\right|^{2}\right. \\
& \left.\quad+\left\langle O\left(k^{2 n}\right), x_{k}-y_{k}\right\rangle\right), \\
& \frac{\partial^{2}\left|P_{k, s}\left(t x_{k}+(1-t) y_{k}, y_{k}\right)\right|}{\partial t^{2}} \\
& =e^{-2 k \operatorname{Im} \Psi\left(t x_{k}+(1-t) y_{k}, y_{k}\right)}\left(\left(\left\langle-2 k \operatorname{Im} \Psi_{x}^{\prime}\left(t x_{k}+(1-t) y_{k}, y_{k}\right), x_{k}-y_{k}\right\rangle\right)^{2}\left|b\left(t x_{k}+(1-t) y_{k}, y_{k}, k\right)\right|^{2}\right. \\
& \quad+\left\langle-2 k \operatorname{Im} \Psi_{x}^{\prime \prime}\left(t x_{k}+(1-t) y_{k}, y_{k}\right)\left(x_{k}-y_{k}\right), x_{k}-y_{k}\right\rangle\left|b\left(t x_{k}+(1-t) y_{k}, y_{k}, k\right)\right|^{2} \\
& \left.\quad+\left\langle-2 k \operatorname{Im} \Psi_{x}^{\prime}\left(t x_{k}+(1-t) y_{k}, y_{k}\right), x_{k}-y_{k}\right\rangle\left\langle O\left(k^{2 n}\right), x_{k}-y_{k}\right\rangle+\left\langle O\left(k^{2 n}\right)\left(x_{k}-y_{k}\right), x_{k}-y_{k}\right\rangle\right), \\
& \frac{\partial P_{k}\left(t x_{k}+(1-t) y_{k}, y_{k}\right)}{\partial t}=\left\langle O\left(k^{2 n}\right), x_{k}-y_{k}\right\rangle, \\
& \frac{\partial^{2} P_{k}\left(t x_{k}+(1-t) y_{k}, y_{k}\right)}{\partial t^{2}}=\left\langle O\left(k^{2 n}\right)\left(x_{k}-y_{k}\right), x_{k}-y_{k}\right\rangle,
\end{aligned}
$$

where $\operatorname{Im} \Psi_{x}^{\prime}(x, y)$ and $\operatorname{Im} \Psi_{x}^{\prime \prime}(x, y)$ denote the derivative and the Hessian of $\operatorname{Im} \Psi(x, y)$ with respect to $x$ respectively. Note that

$$
\left|\left\langle-2 k \operatorname{Im} \Psi_{x}^{\prime}\left(t x_{k}+(1-t) y_{k}, y_{k}\right), x_{k}-y_{k}\right\rangle\right| \leq \frac{1}{c_{0}} k\left|x_{k}-y_{k}\right|^{2} \rightarrow 0 \text { as } k \rightarrow \infty
$$

and

$$
\left\langle-2 k \operatorname{Im} \Psi_{x}^{\prime \prime}\left(t x_{k}+(1-t) y_{k}, y_{k}\right)\left(x_{k}-y_{k}\right), x_{k}-y_{k}\right\rangle<-c_{0} k\left|x_{k}-y_{k}\right|^{2},
$$

where $c_{0}>0$ is a constant independent of $k$. From this observation, (4.21) and (4.24), it is straightforward to see that $\liminf _{k \rightarrow \infty} \frac{f_{k}^{\prime \prime}\left(t_{k}\right)}{\left|x_{k}-y_{k}\right|^{2} k}<0$. From this and (4.22), we get a contradiction.

The theorem follows.

From Theorem 4.6 and Theorem 4.7, we obtain Theorem 4.3 and Kodaira embedding theorem follows then. 


\section{REFERENCES}

[1] R. Berman, B. Berndtsson and J. Sjöstrand, A direct approach to Bergman kernel asymptotics for positive line bundles, Ark. Math. 46 (2008), no. 2,197-217.

[2] L. Boutet de Monvel and J. Sjöstrand, Sur la singularité des noyaux de Bergman et de Szegö, Astérisque, 34-35 (1976), 123-164.

[3] D. Catlin, The Bergman kernel and a theorem of Tian, Analysis and geometry in several complex variables, Katata, Trends in Math, 1-23.

[4] X. Dai, K. Liu and X. Ma, On the asymptotic expansion of Bergman kernel, J. Differential Geom., 72, (2006), no. 1, 1-41.

[5] J.-P. Demailly, Complex analytic and algebraic geometry, available at www-fourier.ujfgrenoble.fr/ demailly/books.html

[6] L. Hörmander, An introduction to complex analysis in several variables, North-Holland mathematical library, NorthHolland Publishing Co., Amsterdam, 7, 1990.

[7] C.-Y. Hsiao, Projections in several complex variables, Mém. Soc. Math. France, Nouv. Sér., 123 (2010), 131 p.

[8] C-Y. Hsiao and G. Marinescu, Asymptotics of spectral function of lower energy forms and Bergman kernel of semi-positive and big line bundles, Communication in Analysis and Geometry, 22 (2014), 1-108 p.

[9] S. Ji and B. Shiffman, Properties of compact complex manifolds carrying closed positive currents, J. Geom. Anal. 3 (1993), no. $1,36-61$.

[10] X. Ma, Geometric quantization on Kähler and symplectic manifolds, Proceedings of the international congress of mathematicians (ICM 2010), Hyderabad, India, August 19-27, 2010. Vol II, 785-810.

[11] X. Ma and G. Marinescu, Holomorphic Morse inequalities and Bergman kernels, Progress in Math., vol. 254, Birkhäuser, Basel, 2007, $422 \mathrm{pp}$.

[12] X. Ma and G. Marinescu, Generalized Bergman kernels on symplectic manifolds, Adv. Math., 217 (2008), no. 4, 17561815.

[13] S. Zelditch, Szegö kernels and a theorem of Tian, Internat. Math. Res. Notices., (1998), no. 6, 317-331.

Institute of Mathematics, Academia Sinica, 6F, Astronomy-Mathematics Building, No.1, Sec.4, Roosevelt ROAD, TAIPEI 10617, TAIWAN

E-mail address: chsiao@math.sinica.edu.tw or chinyu.hsiao@gmail.com 